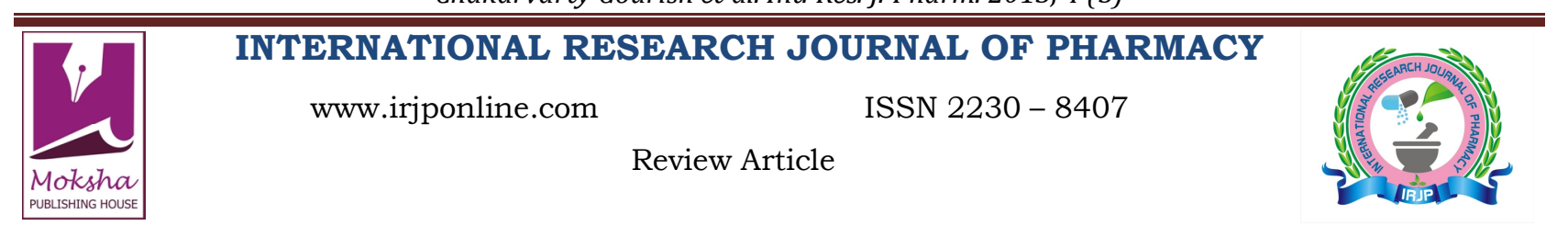

\title{
PROCESS VALIDATION OF SOLID ORAL DOSAGE FORM AND PROCESS VALIDATION GUIDANCE FOR INDUSTRY
}

Chakarvarty Gourish*, Seth Nimrata, Sharma Vishal

Department of Pharmaceutics, Rayat Institute of Pharmacy, Punjab, India

*Corresponding Author Email: gourish.c.sharma@gmail.com

Article Received on: 30/03/13 Revised on: 28/04/13 Approved for publication: 11/05/13

DOI: $10.7897 / 2230-8407.04509$

IRJP is an official publication of Moksha Publishing House. Website: www.mokshaph.com

(C) All rights reserved.

\section{ABSTRACT}

Validation is one of the important steps in achieving and maintaining the quality of the final product. Validation of the individual steps of the processes is called the process validation. Process validation's main objective continues to be the generation of a process which yields a product which meets predetermined quality criteria. It is an important component in the design, prototyping and manufacturing process and one, if done correctly, that can save a considerable amount of time, money and resources. End-product testing by itself does not guarantee the quality of the product. Therefore Quality assurance techniques must be used to build the quality into the product at every step and not just tested for at the end. Process Validation performs this task to build the quality into the product at every step. FDA has released various guidelines for process validation. This guidance incorporates principles and approaches that all manufacturers can use to validate manufacturing processes. FDA considers appropriate elements of process validation for the manufacture of human and animal drugs and biological products, including active pharmaceutical ingredients.

Keywords: Validation, Quality, process, guidance.

\section{INTRODUCTION}

Process validation ensures that a process consistently produces a product that meets its specifications. There are two main reasons for validating a process. The first is to reduce production costs of sorting and rework due to the manufacture of non-conforming products means products that do not meet their specifications. The second is to meet regulatory requirements given by regulatory bodies, such as FDA and to be in compliance with GMP requirements. The prime objective of any pharmaceutical plant is to manufacture products of requisite attribute and quality consistently, at the lowest possible cost. Although validation of drug products have been conducted in the pharmaceutical industry for a long period of time, there is an ever increasing interest in validation owing to their industry's greater emphasis in recent years on assurance of quality and productivity improvement. Validation is everyone's job. It means it is performed in team work. Validation requires team work of all departments like Validation coordinator (protocol, sampling and record), production (facilitating in validation exercise), quality control (validation testing), regulatory affair (dossier), Research and development (critical parameters). Therefore an integrated team approach to process validation that includes expertise from a variety of disciplines (e.g., process engineering, industrial pharmacy, analytical chemistry, microbiology, statistics, manufacturing, and quality assurance) Project plans, along with the full support of senior management, are essential elements for success. It serves to support the underlying definition of validation, which is a systematic approach to identifying, measuring, evaluating, documenting, and re-evaluating series of critical steps in the manufacturing process that require control to ensure a reproducible final drug product. Tablet is a solid pharmaceutical dosage form ${ }^{1}$. The manufacturing of solid dosage forms or drug products involves extensive powder handling. The powder must be blended for uniformity and converted into the dosage form either through compression or encapsulation. Typical requirements include weighing, blending, mixing/granulation areas compression/encapsulation areas, and coating areas ${ }^{2 .}$ In all stages of the product lifecycle, good project management and good archiving that capture scientific knowledge will make the process validation program more effective and efficient.

\section{Plan of validation}

The successful validation is achieved thorough understanding of both the manufacturing process and the product specifications. Validation programme should be clearly defined and documented in a validation master plan (VMP) or other similar equivalent documents. It is a document which summarizes the company's overall philosophy, intentions or approaches that are used for establishing performance adequacy. A validation master plan should be agreed upon by management of the pharmaceutical company. Remember that every process has its own critical steps but may be less critical when compared to the others, so priority and the validation extent should be planned in validation master plan. It is mentioned in GMP guidelines that every critical process should be validated but that does not mean that each and every step should be validated. So it should be planned in validation master plan that which critical steps require validation. The Components of validation master plan includes its objectives and scope. These should be written short, concise and to the point. A brief description, which should be general in nature, should be outlined. The description should not be restrictive, as it is a plan to be loosely followed.

The table of contents for Validation master plan can be written as

1.0 Plan objective

2.0 Plan scope

3.0 Validation approach

4.0 Responsibilities

5.0 Process and facility descriptions

6.0 Systems to be validated

7.0 General acceptance criteria

8.0 Validation schedule

9.0 Personnel and their qualification 
10.0 Supporting programs

11.0 Appendix

\section{Qualification}

Validation and Qualification are essentially components of the same concept. Qualification should be completed before process validation is performed. The process of Qualification is a logical, systematic process and should start from the design phase of the premises and equipments ${ }^{3}$.

Depending on the function and operation of the equipment utility or system. It requires

Installation Qualification (IQ)

Operational Qualification (OQ)

Performance Qualification (PQ)

\section{Installation Qualification (IQ)}

The IQ ensures that all equipment has been installed correctly with applicable inputs (e.g., power or compressed air), all environmental conditions have been met (e.g., temperature or humidity or air quality), all required calibrations have been performed (e.g., pressure gauges or temperature gauges), all safety measures have been implemented and the equipment has been entered into the manufacturer's PM (Preventive Maintenance) and calibration systems to ensure proper maintenance.

\section{Operational Qualification (OQ)}

The OQ demonstrates that the process produces conforming product throughout the range of process inputs, which include process parameters, raw material specifications, production logistics and duplicate sets of equipment where appropriate. Including worst-case combinations of process parameters is critical to demonstrate that the entire range of process parameters will produce acceptable product.

\section{Performance Qualification (PQ)}

The PQ demonstrates that the process consistently produces acceptable product. Often this is interpreted as producing three lots at the nominal process parameters.

\section{Process Validation}

According to USFDA In 1978 "A validated manufacturing process is one which has been proved to do what it purports or is represented to do. The proof of validation is obtained through the collection and evaluation of data, preferably, beginning from the process development phase and continuing through the production phase. Validation necessarily includes process qualification (the qualification of materials, equipment, systems, buildings, personnel), but it also includes the control on the entire process for repeated batches or runs." In 1987 "Process validation is establishing documented evidence which provides a high degree of assurance that a specific process (such as the manufacture of pharmaceutical dosage forms) will consistently produce a product meeting its predetermined specifications and quality characteristics." In 2011"Process validation is defined as the collection and evaluation of data, from the process design stage through commercial production, which establishes scientific evidence that a process is capable of consistently delivering quality product." $4,5,6$

\section{Types of Process Validation}

The various types of process validation are outlined below

\section{Prospective process Validation}

In this type of validation experimental plan called the validation protocol is executed before the process is put to commercial use. Most validation efforts require some degree of prospective experimentation to generate validation support data. This particular type of process validation is normally carried out in connection with the introduction of new drug products and their manufacturing processes ${ }^{7}$.

\section{Concurrent Process Validation}

Concurrent validation is carried out during normal production. The document requirements are same as prospective validation. It is same as that of the prospective validation, except the operating firm will sell the product during qualification runs. The first three production-scale batches must be monitored as comprehensively as possible. The nature and specifications of subsequent in-process and final tests are based on the evaluation of the results of such monitoring. The nature and specifications of subsequent inprocess and final tests are based on the evaluation of the results of such monitoring. This type of validation involves in process monitoring of critical processing steps and product testing, this helps to generate the document evidence to show that the production process is in a state control ${ }^{8}$.

\section{Retrospective Process Validation}

Retrospective validation involves the examination of past experience of production on the assumption that composition, procedures, and equipment remain unchanged; such experience and the results of in-process and final control tests are then evaluated. This type of validation is acceptable only in case of well-established processes, without any change in the composition of the product, operating procedures and Equipment. Retrospective validation is not a quality assurance measure in itself, and it should never be applied to new processes or products. It may be considered in special circumstances only like when validation requirements are first introduced in a company. Retrospective validation may then be useful in establishing the priorities for the validation programme. If the results of a retrospective validation are positive, then this indicates that the process is not in need of immediate attention and may be validated in accordance with the normal schedule. Concurrent validation together with a trend analysis including stability should be carried out to an appropriate extent throughout the life of the product ${ }^{9}$.

\section{Revalidation}

Revalidation is the repetition of a validation process or a part of it. This is carried out when there are any changes or replacements in the formulation or equipment plan and equipment site of location. Revalidation is needed to ensure that changes in the process or in the process environment, whether intentional or unintentional, do not adversely affect process characteristics and product quality. Almost all GMP texts recommend that whenever there are significant changes in the facility, equipment or process, re-validation should be carried out ${ }^{10}$.

\section{Approaches to process validation}

Two basic approaches to the validation of the process itself exist (apart from the qualification of equipment used in production, the calibration of control and measurement instruments, the evaluation of environmental factors, etc.) namely the experimental approach and the approach based on the analysis of historical data ${ }^{11,12}$. 
Stage1: Process Design

The commercial process is defined during this stage based on knowledge gained through development and scale up activities.

Stage2: Process Qualification

During this stage the process design is evaluated to determine if the process is capable of reproducible commercial manufacturing.

Stage3: Continued Process Verification

Ongoing assurance is gained during routine production that the process remains in a state of control.

\section{Critical Factors}

Critical factors which affect effective process validation ${ }^{13,14}$

1. All the critical point of the process should be clearly identified.

2. The process should run using the extremes of the system at the critical points.

3. The quality system should support the validation effort by way of document control, preventive maintenance, calibration, etc.

4. Adequate data are required to provide statistical support to demonstrate product consistency.

\section{Validation Protocol and Report}

A suggested scheme for the validation protocol and subsequent report concerning a particular process is shown below

Part 1.Purpose (the validation) and prerequisites

Part 2.Presentation of the entire process and sub processes, flow diagram, critical steps/risks.

Part 3.Validation protocol, approval

Part 4.Installation qualification, drawings

Part 5.Qualification protocol/report ${ }^{15,16}$

\subsection{Sub process 1}

\subsubsection{Purpose}

5.1.2 Methods/procedures, list of manufacturing methods, SOPs and written procedures, as applicable.

5.1.3 Sampling and testing procedures, accordance criteria (detailed description of, or reference to, established procedures, as described in pharmacopoeias)

5.1.4 Reporting

5.1.4.1 Calibration of test equipment used in the production process

5.1.4.2 Test data (raw data)

5.1.4.3 Results (summary)

5.1.5 Approval and requalification procedure

\subsection{Sub process 2 (same as for Sub process 1)}

5. $n$ Sub process $n$

Part6. Product characteristics, test data from validation batches

Part7. Evaluation, including comparison with the acceptance criteria and recommendations (including frequency of revalidation/requalification)

Part8. Certification (approval)

Part9. If applicable, preparation of an abbreviated version of the validation report for external use, for example by the regulatory authority. The validation protocol and report may also include copies of the product stability report or a summary of it, validation documentation on cleaning, and analytical methods.

\section{FDA Guidance of Process Validation}

The United States Food and Drug Administration (FDA) is responsible for assuring the safety, efficacy, and security of products sold in the USA in the categories of human and veterinary drugs, biological products, medical devices, cosmetics, and products that emit radiation ${ }^{17}$. FDA has issued various guidelines for industry and auditors. FDA published guidance document in 1987, when it was relatively a new concept to industry ${ }^{18}$. In January 2011, the FDA published an updated guidance entitled Guidance for Industry- Process Validation: General Principles and Practices. The new version brings the guidance document by including evolutionary developments, as well as introducing the newest concepts in process validation.

\section{Key changes in new guidance}

The new guidance is a complete rewrite of the old 1987 document. There is very little retained wording from the original, although the general intent of the documents is similar. There are several key points of difference, from the formal definition of process validation, to emphasis on product life cycle and risk management concepts. In the past, process validation emphasis was on collecting large quantities of data leading to a perception of process validation as largely a documentation exercise but the updated approach requires the manufacturer to collect data throughout the product life cycle and evaluate it scientifically and assess if it supports a quality process. The concept of worst-case conditions for process validation was a key theme of the old guidance. This worst case condition has been revised in new guidelines. The worst-case concept is given scant consideration within process validation exercises. Other minor changes include the acknowledgement of some concepts which have gained wide acceptance in industry including

- Integrated team approach - new guidance strongly recommends input in the validation process from a wide range of disciplines, as well as the full support of the senior management

- Process Analytical Technologies (PAT) - the guidance introduces PAT concepts and gives guidance on the role it can play in process validation.

\section{CONCLUSION}

It can be concluded that Pharmaceutical validation and process control are necessary to ensure that drug product will meet pharmaceutical standards for quality, purity, stability and efficacy. It is an important part of among all validation like equipment validation, cleaning validation, vender validation etc. It is a important factor in the quality assurance of pharmaceutical product as the end product testing is not sufficient to assure quality of finished product. It is a key element in assuring that the quality goals are met in compliance with the various guidelines given by GMP and FDA.

\section{REFERENCES}

1. Nash RA and Wachter AH. Pharmaceutical Process Validation; $3^{\text {rd }}$ ed; Marcel Dekker publication.2003: 432 - 434.

2. Levin M, Pharmaceutical process scale-up, Marcel Dekker, Inc. New York. 2002: 313 PMid:11897257

3. Quality Management Systems - Process Validation Guidance by SG3 GHTF/SG3/N99, $2^{\text {nd }}$ ed; 2004. p. 10

4. Guideline on General Principles of Process Validation, FDA, CDRH/CDER; 1987.

5. Federal Food Drug And Cosmetic Act, Title 21 U.S. Code, Section 501 (a) (2) (B). 
6. Rathore S Anurag, Noferi F Joseph, Arling R Edward, Sofer Gail, Watler Peter and Leary O Rhona. Process Validation How Much to Do and When to Do It, BioPharm; 2002.

7. Chao AY, St John Forbes E, Johnson RF, Von Doehren P. Prospective Process Validation. In: I Berry, RA Nash, eds. Pharmaceutical Processing Validation, Marcel Dekker, New York. 1993; 23: 227-248.

8. Nash RA. Process Validation of Pharmaceutical Ingredients. In: Nash RA, Wachter AH. Pharmaceutical Process Validation, An International $3^{\text {rd }}$ ed. Revised and Expanded, Marcel Dekker, New York, 2003; 129: 809-813.

9. Avallone HE. Retrospective Validation, NAPM Meeting, Port Chester, New York. 1983: 29-32.

10. K Kiran and K Kathiresan. Basic Principles of Validation.2005; 1: 3536.

11. FDA, (CDER, CBER, CVM, and ORA), PAT-A Framework for Innovative Pharmaceutical Development, Manufacturing and Quality Assurance, Guidance for Industry; 2004.

12. Gupta GD, Garg R and Aggarwal. Guidelines on General Principles of Validation: Solid, liquid and Sterile dosage forms. www.pharminfo.net.2008; 6: 28-33.
13. James Agalloco, Frederick J Carleton. Validation of Pharmaceutical Processes, $3^{\text {rd }}$ ed, Informa Healthcare USA, Inc., New York; 2008.

14. Bhattacharjee D et al. Industrial applications of process validation in the development and scale up of pharmaceutical tablet dosage form. J Pharm Sci Tech. 2011; 3: 570-574.

15. Lambert J. Validation Guidelines for Pharmaceutical Dosage Forms Health Canada/Health Products and Food Branch Inspectorate, 2004: 715.

16. South African Guidelines for good manufacturing practice, Medicinal Control Council, Pretoria; 1996.

17. US Food and Drug Administration. Guidance for industry. Guidelines on general principles of process validation. Rockville, MD; 1987.

18. FDA/ICH (CDER and CBER) Q10 Pharmaceutical Quality System, guidance for industry; 2009.

Cite this article as:

Chakarvarty Gourish, Seth Nimrata, Sharma Vishal. Process validation of solid oral dosage form and process validation guidance for industry. Int. Res. J. Pharm. 2013; 4(5):36-39 